\title{
REBOTE PARABÓLICO SOBRE UNA SUPERFICIE CURVA CONOCIDA
}

\author{
PARABOLIC BOUNCE ON A SURFACE CURVE KNOWN
}

\section{ALBERTO ANTONIO TIRADO SANABRIA}

En el lanzamiento de proyectiles todo cuerpo describe un movimiento parabólico del tipo cóncavo hacia abajo, por efecto de la gravedad terrestre y la trayectoria seguida ocurre desde que se lanza hasta que choca contra una superficie. Si esta es una curva y el choque es elástico o casi elástico entonces el ángulo o dirección de salida resulta hasta ahora desconocida en el mundo de la física clásica. En este trabajo se expone la deducción de una ecuación que permite conocer la dirección de salida de uno o varios movimientos parabólicos originados como consecuencia de uno o varios rebotes, de un cuerpo, sobre una superficie curva representada por una función del plano cartesiano.El cálculo de la dirección de rebote está fundamentada en el análisis que se realiza en el rebote de una partícula sobre una superficie recta inclinada y donde se plantea ampliar el rebote, hacia superficies no rectas; luego esta posibilidad puede tener aplicaciones de índole académico en los estudios generales de ingeniería, así como de la vida y el campo de trabajo. La investigación tiene como objetivo descubrir los nuevos movimientos parabólicos posibles, generados ante el rebote de un cuerpo elástico sobre una superficie curva. Sin embargo, este cálculo y la relación que se expone resulta un preliminar para la variedad de situaciones que se pueden generar en movimientos parabólicos de cuerpos que colisionan contra una superficie dada conocida.

PALABRAS CLAVE: rebote parabólico, superficie curva conocida.

KEYWORDS: Parabolic rebound, known curved surface.

ABSTRACT

In the launching of projectiles, everybody describes a parabolic movement of the concave type downwards, by the effect of terrestrial gravity and the trajectory followed occurs from the moment it starts until it hits or encounters a surface. If this is a curve and the shock is elastic or almost elastic, then the angle or direction of departure is hitherto unknown in the world of classical physics. In this work, the deduction of an equation that allows to know the exit direction of one or more parabolic movements originated as a result of one or more bounces of a body on a curved surface represented by a continuous function of the Cartesian plane is exposed.

The calculation of the direction of rebound is based on the analysis that is made of the rebound of a particle on a straight inclined surface and where it is proposed to extend the bounce, towards non-straight surfaces; then this new possibility may have applications of an academic nature in the general engineering studies, as well as life and the field of work. The objective of the research is to discover the new possible parabolic movements generated by the rebound of an elastic body on a curved surface. However, this calculation and the relationship that is exposed is a preliminary for the variety of situations that can be generated in parabolic movements of bodies that collide against a given known surface. 
INTRODUCCIÓN

Para un proyectil en una trayectoria parabólica que choca contra cualquier superficie sólida impenetrable, puede ocurrir según las características del cuerpo, un rebote que será dependiente de la forma de la superficie. Si la superficie es una recta y el choque es casi elástico, entonces el ángulo de incidencia será igual al del rebote, lo que genera uno o varios movimientos parabólicos consecuentes y conocidos en los casos de: rebote sobre una línea recta vertical, sobre una línea recta horizontal, y sobre una línea recta inclinada (Tirado, 2018). En donde quedó como reto al lector, el rebote parabólico de una partícula sobre una superficie curva.

En este trabajo se expone la deducción de una sencilla ecuación que permite conocer la dirección de salida de uno o varios movimientos parabólicos originados como consecuencia de uno o varios rebotes de un cuerpo sobre una curva dada, siempre y cuando ésta curva esté representada por una función del plano cartesiano, sea ésta algebraica o trascendente. Ahora, cuando la partícula regrese a un nuevo contacto con la superficie, por efecto de la gravedad terrestre, se puede conocer por integración la longitud de arco entre rebotes de la superficie que recorre la partícula.

La deducción de la nueva dirección de salida de la partícula al rebotar sobre una superficie, determina una ecuación parabólica que puede o no coincidir nuevamente con la función que representa a la superficie en donde choca la partícula. Entonces, por análisis algebraico, se debe deducir este encuentro para el estudio del siguiente contacto y posible siguiente rebote. Recordando que el rebote sobre superficies rectas se estudia en la mecánica avanzada con los conocimientos de la conservación de la energía y la cantidad del movimiento; tema conocido como cinética de la partícula (Berr y Russel, 1999).

DESARROLLO MOVIMIENTO EN EL PLANO DEL TIPO PARABÓLICO En un movimiento en el plano del tipo parabólico (lanzamiento de proyectiles con la aceleración horizontal constante en cero y la aceleración vertical constante en la gravedad terrestre) que finalice sobre una superficie en línea recta, se le puede calcular el ángulo de choque final o ángulo de incidencia por análisis del vector velocidad final en función del parámetro tiempo. Todo ello, por medio de ecuaciones individuales que analizan el comportamiento de la partícula en cada eje por separado. Es decir, el estudio de un movimiento en el plano del tipo parabólico utiliza el sistema de ecuaciones paramétricas para cada eje coordenado en función del tiempo.

- Eje horizontal:

1. $V_{f x}=V_{o x}$

2. $X=X_{o}+V_{o x} t$

- Eje vertical:

1. $V_{f y}=V_{o y}+g t$

2. $Y=Y_{o}+V_{\text {oy }} t+\frac{1}{2} g t^{2}$

- Ecuación de la parábola:

$$
Y=Y_{o}+X \tan \theta+\frac{1}{2}\left(\frac{g X^{2} \sec ^{2} \theta}{V_{o}^{2}}\right)
$$

Siempre que el punto de partida del lanzamiento sea el origen coordenado $(0,0)$ y el ángulo de lanzamiento sea con respecto a la horizontal.

- El vector velocidad final es:

$$
V f=V_{f x}+V_{f y}
$$

- El ángulo $\alpha$ es igual a la: $\tan ^{-1}\left(\frac{V_{f y}}{V_{f x}}\right)$, como ángulo de incidencia sobre una superficie cualquiera sea ésta donde termine el movimiento parabólico.

\section{MOVIMIENTO DE REBOTE CONTRA UNA SUPERFICIE}

Si el objeto lanzado rebota de la superficie de tal forma que no exista deslizamiento o rotación del mismo, el tiempo de contacto sea ínfimo, el choque se considere aproximadamente elástico y el objeto tenga capacidad elástica para rebotar, entonces el ángulo de salida del rebote sobre la superficie (final del lanzamiento) será igual:

a. el ángulo de incidencia para superficies rectas horizontales $y / o$ verticales, $\alpha=\beta$. Ecuación 1.

b. El ángulo de incidencia menos dos veces el ángulo de la línea recta inclinada, en rebote "hacia abajo", $\alpha=\beta-2 \theta$. Ecuación 2.

c. El ángulo de incidencia más dos veces el ángulo de la línea recta inclinada en rebote "hacia arriba", $\alpha=\beta+2 \theta$. Ecuación 3 (Tirado 2018).

\section{REBOTE PARABÓLICO SOBRE UNA SUPERFICIE CURVA}

Para poder determinar el rebote parabólico sobre una superficie curva, se deben cumplir los siguientes parámetros: 
a. La superficie donde rebota el cuerpo que incide con un movimiento parabólico, debe estar representada por una función del plano coordenado conocido.

b. Esta función debe ser derivable y continua en el intervalo de posibles contactos con la trayectoria parabólica del cuerpo a rebotar sobre ella. Por la definición de la derivada como función que representa la recta tangente a la función derivable, y del hecho que toda evaluación en un punto dado "X1" es la pendiente de esta recta (Benítez, 2006).

Cumplidas estas condiciones, el rebote sobre una superficie conocida estará fundamentado en el rebote sobre una superficie recta inclinada, debido a que el punto de contacto del cuerpo con la superficie se considerará común con la recta tangente a la curva en ese punto, el cual se obtiene por la derivada de la función. Es decir, la pendiente de la recta tangente de la función que representa la superficie de contacto en el punto donde se encuentra con el cuerpo en su movimiento parabólico, nos permite conocer el ángulo de inclinación en donde va a rebotar el cuerpo según es mostrado en la Figura 1.

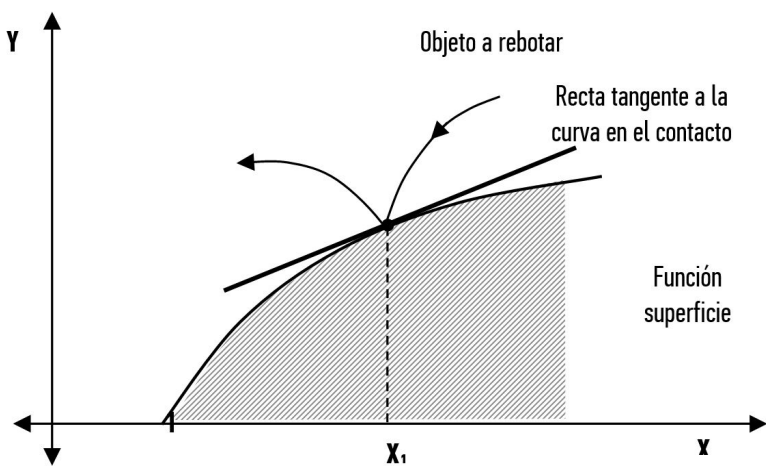

Figura 1. Ejemplo de un movimiento parabólico en contacto con una superficie curva representada por la función trascendente, logaritmo natural: $Y=\operatorname{Ln}(X)$

A partir de este rebote, se calcula el nuevo ángulo de salida o de rebote del cuerpo impactante sobre la superficie, por las ecuaciones del rebote parabólico sobre una línea recta inclinada. Éste ángulo y el punto de contacto, como punto inicial del nuevo movimiento parabólico, permitirán obtener una nueva ecuación de movimiento tipo parabólico para el cuerpo; al incluir la información de éste punto, su orientación y la del ángulo nuevo de rebote según se muestra en la Figura 2.

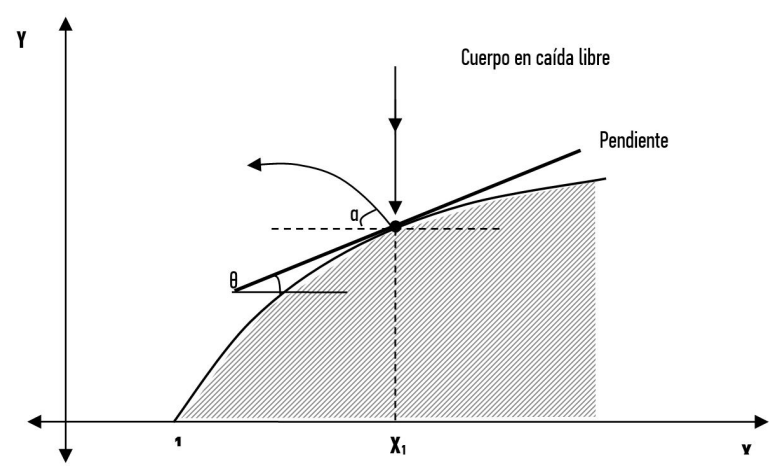

Figura 2. Con el ángulo de la inclinación como: $\theta=\tan \left(Y^{\prime}\left(X_{1}\right)\right)$ y el ángulo de rebote como: $a=\beta-2 \theta$. Es decir: $a=90^{\circ}-2 \theta$.

La ecuación del nuevo movimiento parabólico ${ }^{\text {es }} Y=Y_{o}-\left(X-X_{o}\right) \tan (\alpha)-\frac{\left(4,9\left(X-X_{O}\right)^{2} \sec ^{2}(\alpha)\right)}{V_{O}^{2}}$

Donde $\mathrm{V}_{\mathrm{o}}$ es la rapidez inicial del rebote condicionada a la rapidez final del contacto. $\left(\mathrm{X}_{\mathrm{o}}, \mathrm{Y}_{\mathrm{o}}\right)$ es el punto inicial del nuevo movimiento parabólico en el rebote y el signo negativo en el tercer término se refiere a la orientación hacia abajo que sigue el cuerpo por efecto de la gravedad terrestre.

Seguidamente se verifica si el cuerpo y la superficie curva vuelven a coincidir en un punto de contacto, y por ende en el próximo rebote. Para esto, se igualan las ecuaciones del nuevo movimiento parabólico con la ecuación representativa de la superficie curva, lo que origina tres situaciones de complejidad matemática diferente.

- Situación 1: la nueva ecuación parabólica del cuerpo que rebota y la ecuación que representa la curva no tiene solución, cuando no existe un resultado ni geométrico ni algebraico al igualarse. Lo que implica que no existirá un nuevo contacto entre ambas y por ende no ocurrirá un nuevo rebote.

- Situación 2: la solución de la igualdad de la ecuación potencial parabólica con una ecuación del tipo algebraica representativa de la superficie curva tiene una solución exacta o sólo tiene solución por aproximación de raíces poli nómicas (Método de Newton-Rapson). Lo que permite encontrar el nuevo punto de contacto sin o con un mínimo de error. Luego a partir de este conocimiento se halla el nuevo ángulo de rebote y la nueva ecuación parabólica del cuerpo según se muestra en la Figura 3. 


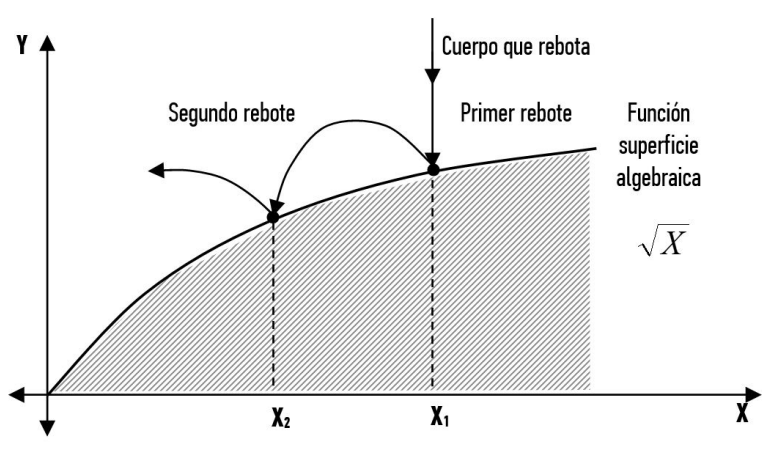

Figura 3. El cuerpo que rebota regresa a la superficie curva representada por una función algebraica del plano cartesiano, en el punto $X_{2}$.

- Situación 3: la solución de la igualdad de la ecuación potencial parabólica con una ecuación del tipo transcendente representativa de la superficie curva tiene una solución exacta o sólo tiene solución geométrica por medio del uso de una calculadora con función de gráfica, o a través de técnicas matemáticas de gráficas de relaciones en el plano. Lo que permite encontrar la solución del punto de contacto con un mínimo de error y por ende, se puede calcular el nuevo ángulo de rebote y la nueva ecuación parabólica del cuerpo que rebota.

Luego si se conocen los dos puntos de contacto en el eje "X": $\left(\mathrm{x}_{1} \mathrm{y} \mathrm{x}_{2}\right)$ donde el cuerpo rebota y retorna a la superficie conocida como una función del plano, entonces por aplicación de la integración se puede conocer el arco de curva "desplazado" por el cuerpo sobre su vuelo sobre la superficie, al realizar la integración para cálculo de arco de la ecuación de la superficie entre los puntos de contacto ya estimados según se muestra en la Figura 4.

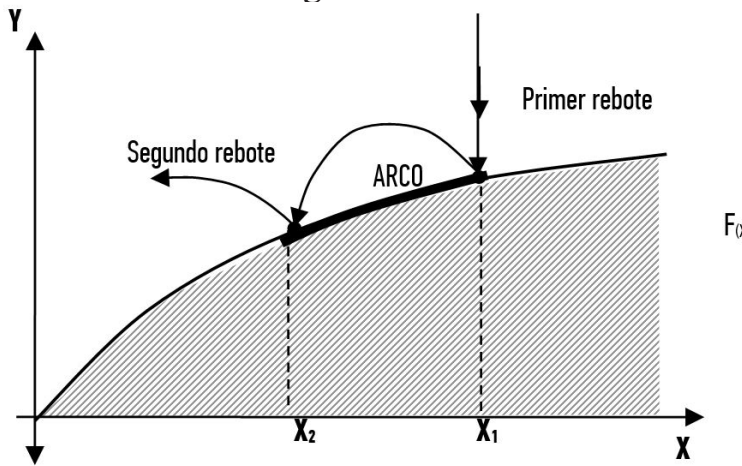

Figura 4. El cuerpo después de dos rebotes sobre la superficie curva.

El cálculo del arco desplazado por el cuerpo que rebota, medido en distancia sobre la superficie curva, viene expresado para su cálculo por la relación:
Finalmente todo el proceso se reitera en un círculo que culmina con la situación 1 , en donde ya no existirá un nuevo contacto entre el cuerpo que rebota y la superficie cuerva representada por una ecuación matemática (Purcell, Varberg y Rigdon, 2001).

\section{MATERIALES Y MÉTODOS}

Las ecuaciones deducidas en este trabajo pueden ser validadas en experimentos de rebote parabólico de cuerpos esféricos con capacidad de rebotar y el uso de una superficie diseñada de tal forma que esté representada por una función del plano cartesiano. El experimento en sí consistiría en dejar caer pelotas sobre esta superficie y que esta permita que se dejen huellas en los contactos y anotar el punto de contacto (primer rebote) y el segundo contacto (segundo rebote). Un esquema posible fue ilustrado en la Figura 4.

Con estos datos se puede calcular por análisis de un movimiento parabólico el ángulo de rebote o de salida " $\alpha$ " a partir del cálculo de los puntos de contactos y compararlo con el de la ecuación 2 para validar está deducción y utilidad sobre superficies curvas. En este sentido los materiales requeridos serán los siguientes:

- Superficie de madera o plástico diseñada con la forma de una función del plano.

- Papel carbón y blanco para tomar notas de las impresiones de los cuerpos al colisionar con la superficie y rebotar en ella.

- Varias pelotas de plástico, metal y de goma, como cuerpos a rebotar.

- Calculadora científica y juego de escuadras.

\section{RESULTADOS Y DISCUSIÓN EN EJEMPLOS DADOS} EJEMPLO 1

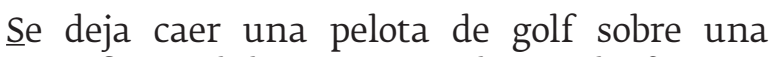
superficie sólida representada por la función transcendente $\mathrm{Y}=\mathrm{Ln}(\mathrm{X})$ en metros desde una altura de 50 centímetros y se conoce que en el contacto, la pelota de golf conserva su rapidez al rebotar de dicha superficie (se asumió choque elástico). Hallar la distancia de arco total que recorre la pelota sobre la superficie después de rebotar y regresar a un segundo contacto, si el contacto inicial es en $\left(\mathrm{X}_{1}=3 \mathrm{~m}\right)$. Ver Figura 5.

RESPUESTA

a. Velocidad y ángulo del primer contacto: $V_{f}=0+2 g(0,5) \Rightarrow V f=-3,13 \hat{j}$

En el punto $X_{1}=3 \mathrm{~m}$, rapidez de salida $3,13 \mathrm{~m} / \mathrm{s}$ 


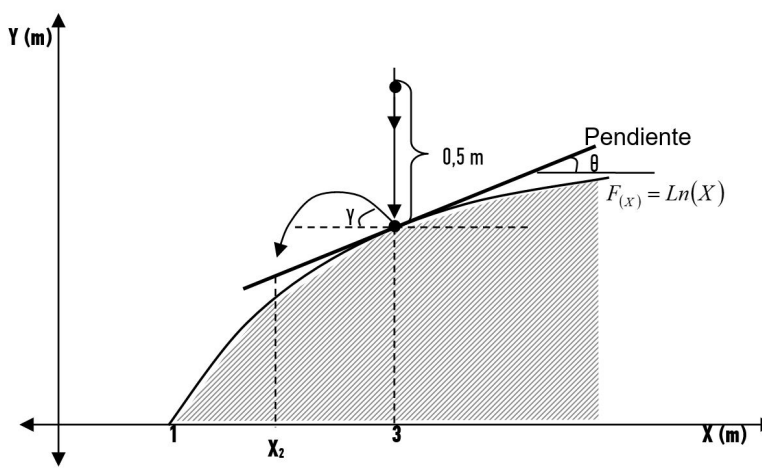

Figura 5. Ejemplo 1, de un cuerpo que rebota después de caer libremente desde una altura de 0,5 metros sobre una superficie representada por la función $\mathrm{y}=\operatorname{Ln}(\mathrm{x})$.

b. Cálculo del ángulo de salida del primer rebote: La pendiente de la recta tangente a la curva en el punto $\mathrm{X}_{1}=3$ es: $Y=1 / X$, $\mathrm{m}=0,3333$ y la inclinación de esta recta es 18,435․ El ángulo de salida será: 90 $2(18,435)=53,13^{\circ}$.

c. La nueva ecuación parabólica del cuerpo será:

$Y=\operatorname{Ln}(3)-(X-3) \operatorname{Tan}\left(53,13^{\circ}\right)-\left(\frac{4,9(X-3)^{2}\left(\operatorname{Sec}^{2}\left(53,13^{\circ}\right)\right)}{9,8}\right)$

d. Cálculo del nuevo punto de contacto por igualación de la ecuación parabólica con la ecuación superficie. La solución es estrictamente geométrica, con $\mathrm{X}_{2}=1,678 \mathrm{~m}$.

e. Finalmente el arco recorrido por el cuerpo en sus dos contactos con la superficie curva resulta de la integración de:

$A R C O=\int_{1,678}^{3} \sqrt{1+\left(\operatorname{Ln}^{\prime}(X)\right)^{2}} d X=\int_{1,678}^{3} \sqrt{1+\frac{1}{X^{2}}} d X$

El resultado de esta integral da como resultado el valor del arco desplazado por el cuerpo en su rebote de: $2,835-1,388=1,448 \mathrm{~m}$.

EJEMPLO 2

Se deja caer una esfera plástica en el punto $\mathrm{X}=$ 1,8 rad sobre una superficie representada por la función $\mathrm{Y}=\mathrm{Sen}(\mathrm{x})$, si la pelota rebota a rapidez de $3 \mathrm{~m} / \mathrm{s}$ según se muestra en la Figura 6. Calcule el arco desplazado entre el primer y segundo contacto con la superficie.

RESPUESTA

Elángulo de incidencia con respecto a la horizontal es $\beta=90^{\circ}$. El punto de contacto es 1,8 radianes $\left(103,14^{\circ}\right)$. La recta tangente a la función $\mathrm{Y}=\operatorname{Sen}(\mathrm{x})$ en el punto tiene por pendiente $\operatorname{Cos}\left(103,14^{\circ}\right)=-$ 0,2273 . Su ángulo es: $\theta=\operatorname{Tan}^{-1}(0,2273)=12,81^{\circ}$.

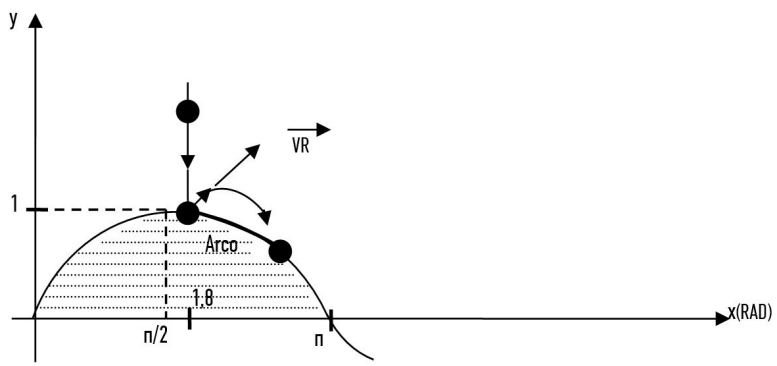

Figura 6. Un cuerpo que rebota después de caer libremente sobre una superficie representada por la función: $Y=\operatorname{Sen}(x)$.

Por la ecuación 4.3.1, el ángulo del rebote es: $\alpha=$ $90^{\circ}-2(12,81)=64,4^{\circ}$.

La parábola de este rebote parabólico tendrá por ecuación:

$Y=\operatorname{Sen}(103,14)-(X-1,8) T \operatorname{Tan}\left(64,4^{\circ}\right)-$

$-\left(\frac{4,9(X-1,8)^{2}\left(\sec ^{2}\left(64,4^{\circ}\right)\right)}{9}\right)$

Cálculo del nuevo punto de contacto por igualación de la ecuación parabólica con la ecuación superficie. La solución es estrictamente geométrica y el nuevo punto de contacto es $\mathrm{X}_{2}=2,73$ rad. (Queda a la confirmación por el lector con el uso de un software de gráficas de relaciones).

El arco de curva sale de la relación:

$$
A R C O=\int_{1,8}^{2,73} \sqrt{1+(\operatorname{Cos}(X))^{2}} d X .
$$

La distancia desplazada es de 1,105 m.

\section{CONCLUSIONES Y RECOMENDACIONES CONCLUSIONES}

El estudio del movimiento en el plano del tipo parabólico es fundamental en el área de la física básica, considerando que casi todos los cuerpos no autopropulsados que lanzamos al aire con una inclinación inicial diferente de los noventa grados, tiene este comportamiento para todo su tiempo de vuelo o tiempo en que el cuerpo regresa a una superficie dada. El estudio del rebote parabólico de un cuerpo con capacidad de rebotar sobre una superficie curva, representada por una ecuación matemática de una función continua, sea transcendente o algebraica, es una consecuencia de la utilidad de las relaciones desarrolladas en el estudio del rebote parabólico sobre una línea recta inclinada, con las aplicaciones matemáticas conocidas de resolver raíces poli nómicas y/o puntos de cruce por medio de asistentes computarizados de gráfica de relaciones. Finalmente, este trabajo abarca el cálculo de la longitud de arco de 
curva que desplaza el cuerpo que rebota por las técnicas de integración conocidas. El estudio del nuevo movimiento parabólico que se genera, en vista que las aceleraciones tanto para el eje horizontal como para el eje vertical permanecen constantes, es posible obteniéndose resultados muy cercanos a la realidad física del comportamiento de un cuerpo que rebota sobre una superficie a la que se le pueda representar por una función del plano coordenado.

En función de lo presentado, se puede decir que el ciclo de cálculos de resultados por iteración desarrollados en este artículo para el cálculo de la dirección que sigue un cuerpo después de un rebote sobre una superficie curva conocida, representan una apertura de opciones para el estudio de las variables y las constantes del movimiento parabólico. Ello genera un abanico de datos e incógnitas a utilizar en este tema; que de desarrollarse corresponde a una aplicación de la derivada para los cursos de las carreras que contengan la
Matemáticas I con esta temática; y una aplicación avanzada en la cátedra de métodos numéricos en las carreras de ingeniería. En vista de que el rebote parabólico sobre una superficie curva representada por una función del plano coordenado es un conocimiento nuevo, se recomienda al lector que analice estudios que demuestren estos resultados y su aplicación académica.

\section{REFERENCIAS BIBLIOGRÁFICAS}

Benítez, R. (2006). Cálculo Diferencial para ciencias básicas e ingeniería. México: Editorial Trillas.

Berr, F., y Russel, E. (1999). Mecánica vectorial para ingenieros. Dinámica. Sexta edición. México: Editorial Mc Graw Hill.

Purcell, E., Varberg, D., y Rigdon, S. (2001). Cálculo. Octava edición. México: Editorial Prentice Hall.

Tirado, A. (2018). El rebote parabólico, sobre una superficie recta inclinada. Paradigma, 30, (1), p. $112-124$. 